\title{
ASL sign lowering as undershoot: A corpus study ${ }^{1}$
}

\author{
Kevin Russell, Erin Wilkinson, and Terry Janzen \\ University of Manitoba \\ revised version - June 15, 2011
}

\begin{abstract}
American Sign Language (ASL) signs that are located on the forehead in their canonical form are often articulated lower during natural signing. Previous studies have examined this phenomenon from a phonetic perspective, treating it as a form of undershoot, and from a variationist sociolinguistic perspective, treating it as a categorical process. This study sees if the findings and explanations of these studies can be extended to the lowering of signs formed in locations other than the forehead. In a corpus of natural conversational signing from six signers, we measure the vertical displacement of over 3000 tokens of signs canonically formed at the face, head, or neck. While there is some apparent evidence for a categorical lowering process in a minority of signs and considerable evidence for undershoot, neither alone can explain the full range of displacement patterns across all signs and locations. Undershoot must be carefully planned and controlled: no matter how sloppy the signing, signers systematically avoid contacting their eyes. The results can be explained if there is a somatosensory forward-modelling mechanism that can veto undesirable gestural scores and whose decisions are incorporated during learning into the phonological distributions representing the locations of signs.
\end{abstract}

\section{Introduction}

This paper considers whether the phenomenon of sign lowering in American Sign Language (ASL) is a categorical process or whether, as suggested by the work of sign-language phoneticians (e.g., Mauk 2003), it might simply be the result of the kind of articulatory undershoot that is well documented in spoken languages.

Undershoot occurs when an articulator executing a gesture fails to make it all the way to its target. In spoken language, this can occur when, for example, the pitch of the voice doesn't make it all the way down to a low tone sandwiched too tightly between two high tones, when the tongue tip doesn't actually contact 


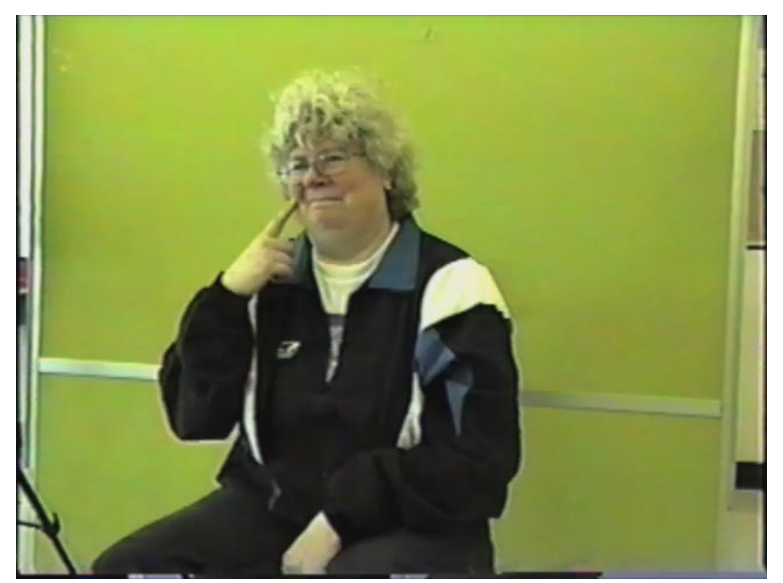

Figure 1: The highest point reached by the index finger for one token of the sign THINK. The canonical location for THINK (when signed with the right hand) is on the forehead over the right eye.

the alveolar ridge during the tap in a word like water, or (to steal an example from Pierrehumbert (2003)) when the $[\mathrm{u}]$ in the first syllable of the word Trudy doesn't get all the way to the back of the vowel space because the tongue body is getting a head start on the following front vowel [i].

The idea of explaining vowel coarticulation with targets has evolved a lot since first proposed by Öhman (1966). The most popular current explanations for the kind of undershoot we see in Trudy, embodied in theories like Articulatory Phonology (Browman \& Goldstein 1992), is that it is caused by two or more targets (or entire gestures) overlapping in time. The tongue body is almost literally being pulled in two directions at the end of the /u/ in Trudy: the target related to [u] wants the tongue body further back, the target for [i] - which is already in force before the end of the $[\mathrm{u}]$ - wants the tongue body further forward, resulting in a half-hearted compromise between the two and causing the tongue to undershooting the backness target. This kind of undershoot is expected to occur more often in fast speech, where there is more overlap between gestures. Another possible explanation of (some cases of) undershoot is that the magnitude of a gesture is simply decreased (less effort is put into reaching the target) for reasons unrelated to gestural overlap. All remarks made about the nature of undershoot in the discussion section of this paper apply equally to either conceptualization.

Sign lowering in ASL is a phenomenon that seems tailor-made for an explanation in terms of articulatory undershoot. Signs that are made at one location on the face in their canonical form are often realized at a lower location, especially in faster conversational signing, and signs which canonically contact the face are often realized without contact. Signs that are canonically made at the level of the signer's forehead seem especially subject to lowering. For example, the sign THINK is canonically made with by the signer's 
index finger contacting mid-way up their forehead approximately above they eye. Figure 1 shows one noncanonical lowered realization, where the signer's index finger did not make it higher than her cheek before contacting. ${ }^{2}$

This process of sign lowering has been studied from two different disciplinary perspectives by researchers within the traditions of experimental phonetics and variationist sociolinguistics. We will briefly summarize the findings of each in turn.

\subsection{Lowering studied by phoneticians}

ASL sign lowering has been studied from within the paradigm of experimental phonetics by Mauk et al. (2008), Mauk \& Tyrone (2008), and Tyrone \& Mauk (2010). These studies all used optical tracking systems (either Vicon or Optotrak) for their data collection, attaching markers (infrared-emitting diodes or reflective disks) to various parts of the signers' bodies (e.g., forehead, shoulders, elbows, pinky finger) and tracking their exact locations in 3D space with infrared cameras.

In all three studies, the signers were asked to vary their rate of signing. The target signs were all canonically formed at the forehead (like WONDER, FATHER, or KNOW) and the vertical location of the target signs' neighbours was manipulated: sometimes high (like SMART or pointing to a high location), sometimes low (in neutral space like RIGHT or CHILDREN, at the chin like BITTER, or at the chest like ME). They found that the vertical position reached during the forehead sign was affected by:

- signing rate. Faster signing makes lowering more likely.

- location of the neighbouring signs. The target sign is more likely to lower when its neighbours are lower signs, like RIGHT or ME, than when its neighbours are higher signs, like SEE or BITTER.

- phrase-initial or phrase-final position. There is a complex pattern of interactions with other factors across signers, but generally phrase-final signs lower more than phrase-initial.

These studies explicitly treat sign lowering as a form of undershoot, offering a simple, appealing explanation of the process that ties it in with other demonstrations that undershoot is at work in the phonetics of ASL (e.g., Cheek 2001a,b; Cormier 2002). Although the authors never claim that their results generalize to all cases of lowering in ASL, even to all cases of lowering of forehead signs, it is worth seeing how far an undershoot explanation can be generalized. 


\subsection{Lowering studied by variationists}

Sign lowering has also been studied from the position of variationist sociolinguistics, notably by Lucas et al. (2002) on American Sign Language and by Schembri et al. (2009) on Australian Sign Language and New Zealand Sign Language.

Both studies recorded signers from a wide range of social groups (age, class, educational background, etc.) in informal conversations. Tokens of signs canonically made on the forehead were binarily coded as either lowered or non-lowered, based on whether their vertical peak was above or below a dividing line. These researchers do not explicitly claim that lowering is a categorical process, but the methodology of logistic regression forces them to analyze their results as if it were.

Both studies found a number of relationships between sign lowering and the signer's membership in social categories - though this was one of the main motivations of their studies, we will not dwell on it here. The non-social influences on sign lowering include some that are striking similar to those of the phoneticians discussed in the last section:

- location of the preceding and following signs. Lower neighbours makes lowering the target sign more likely.

- lexical category. For example, ASL grammatical function signs are more likely to lower than nouns and verbs. In New Zealand Sign Language, verbs are more likely to lower than nouns.

- lexical frequency. For example, in New Zealand Sign Language, high-frequency verbs are more likely to lower than low-frequency verbs, though the situation is reversed for nouns.

- pre-pausal position.

(Not all of these were independently significant in all three languages, and there were some interactions between them.)

\subsection{Are they studying the same phenomenon?}

In some respect, the differences between the lists of influences found in these studies reflect what the researchers were able to or chose to study. The variationist studies have found that nouns and verbs behave differently, while the phonetic studies have used too few sign types to compare lexical categories. The phoneticians have found that signing rate matters, while the variationists had no way to control signers' spontaneous rate of production. 
But apart from these minor differences forced by methodological choices, the list of influences arising from the two kinds of studies are strikingly similar. Not only do the (non-social) influences found in the variationist studies overlap a great deal with the set found by the sign-language phoneticians, they are also quite similar to those known to influence undershoot in spoken languages. This forces us to ask whether the two strands of research are studying the same thing. The phoneticians are measuring vertical displacement continuously, as if lowering were a gradient process. The variationists are measuring vertical displacement with a (perhaps artificial) dichotomization, as if lowering were a categorical process. Apart from those fundamental choices of how to measure, are they in fact measuring the same phenomenon?

It would be tempting to think so. The phonetic studies showed there clearly is (at least mild amounts) of gradient undershoot for forehead signs. It would be a simpler account if we could extend this and treat all cases of sign lowering as a single gradient process. The variationist studies could still have obtained the findings they did if lowering were a gradient process - artificially dichotomizing a gradient continuum into "lowered" and "not lowered" would misrepresent the nature of the process and throw away valuable information, but with thousands of data points even an artificially dichotomized measure will still reveal many of the patterns influencing the magnitude of the process (including those involving social factors).

On the other hand, there are many examples of sign lowering that are difficult to think of as being undershoot. When a signer producing THINK slowly and deliberately places her index finger at her chinline and leaves it there for a substantial fraction of a second, it is hard to explain this as just another example of a signer under time constraints aiming for her forehead and missing. This suggests that there may be either multiple variants for each lexical item or a categorical sign lowering process resulting in two or more discrete target locations, each of which may be mildly undershot.

The current study is an attempt to combine the strengths of the phonetic and the variationist approaches in trying to get at the fundamental nature of the lowering process. As in the variationist studies, our data come from natural discourse - spontaneous, informal conversations, rather than from multiple repetitions of unnatural phrases in the lab by signers wearing cumbersome equipment - and we are able to consider a wide range of sign types/lexemes, though unlike the variationists we do not study enough signers to be able to make any claims about the influence of social categories on sign lowering. As in the phonetic studies, our measurements are continuous (rather than possibly artificially dichotomized), though they are a good deal less precise than the 3D measurements produced by infrared optical tracking. 


\section{Method}

The videos on which the project is based are part of a corpus of conversational ASL signing recorded by Terry Janzen in 2000. The signers were Deaf adults between the ages 18 and 50. Each learned ASL as their first language, beginning by the age of five at the latest and usually in infancy. All consider themselves, and are considered by others, as members of the Winnipeg Deaf community. Ten signers were recorded for the initial corpus. Six of those are analyzed in this paper: two women and four men, all right-handed.

Each signer engaged in informal conversation with an interviewer who was also a Deaf native signer. The interviewer had a set of general topics and questions that could be used if the conversation ever stalled - this was seldom necessary. The interviewer sat to the left of the camera, so signers' faces are usually angled slightly to their right. Both signers and interviewers were video-taped by separate VHS cameras (in NTSC format) at 30 frames per second and digitized onto computer with a resolution of 640 by 480 pixels. Only the recordings of the interviewees are analyzed here.

We chose to analyze lexical signs that are made with reference to a location on the head or neck. Most of the signs actually contact a location on the head or neck in their canonical forms, such as KNOW and NOT, though some do not, such as WHY or REASON. For purely practical reasons, we did not analyze signs like GUESS that are made at the same general height but are not made with respect to any specific landmark on the face that can be reliably identified and measured as the canonical location.

For each of these signs that had at least two tokens in the videos, we determined the "canonical" place of articulation of the contact or near-contact, i.e., that used in the most carefully articulated realizations of the most conservative versions of those signs that are still used at least occasionally by native signers in Winnipeg in the same age range as the signers in the videos. (In fact, almost all the signs had at least one token that reached this canonical location even in the portion of the corpus we analyzed.) These are also almost always the citation forms that signers will offer when asked for signs. For each place of articulation, we determined:

a) the passive articulator $(P A)$ : the location/landmark on the face that is contacted or approached.

b) the active articulator $(A A)$ : the part of the hand that contacts or comes closest to contacting. This is usually one of the selected fingers, in the sense of Mandel (1981), but not always.

We defined the PAs and AAs to be as small as possible so that they could be reliably located and measured in a comparable way across all tokens of a sign. For example, for the sign THINK, the AA is the the tip or 
the top half of the front of the outermost segment of the index finger, and the PA is a spot vertically mid-way up the forehead and horizontally over the centre of the right eye (for tokens made with the right hand).

For each token of each of these signs in the videos, the rough interval containing the sign was marked using the annotation software ELAN (Sloetjes et al. 2006). Following this, a research assistant identified for each token the exact frame of the video where the active and the passive articulator were closest to each other and marked this with another ELAN annotation. For cases where contact lasted for several video frames, the first frame was chosen. For signs that were reduplicated or involved some other repetitive motion, only the first contact or approach was used. For alternating two-handed signs, such as DESPERATE, the first contact/approach by either hand was used. For symmetrical two-handed signs, such as DEER, the first contact/approach by the signer's right hand was used. For the remainder of this paper, we will use the term attainment point to refer to the position of the active articulator in the frame so identified.

For each token, the frame of the attainment point was saved as PNG graphics file and, using custom software, the canonical position of the PA and the actual position of the AA were marked with a mouse. The coordinates (in pixels) were automatically logged. For each token, the positions of the signer's two eyes, tip of nose, and tip of chin were similarly recorded, to be used for rough normalization across different signers and different postures of the same signer. Because signers very often had their head tilted, pixel coordinates were transformed into a coordinate system rotated around the canonical PA by the same angle as between the two eyes. Tokens articulated with the left hand were reflected across the rotated vertical axis and thereafter treated as though they had been produced by the right hand. The vertical displacement for each token was taken to be the $y$ coordinate of its attainment point in this rotated coordinate space - since 0 is by definition the vertical level of the canonical PA, a negative $y$ coordinate for the attainment point means the sign has been lowered. Each token was also coded for whether the AA actually contacted any part of the signer's body at the attainment point and its lexical category. The lexical category - noun, verb, adjective, negative verb, and other - was coded only for tokens where the lexeme was unambiguous (e.g., FATHER) or, for ambiguous lexemes (e.g., EAT/FOOD), only if the category was clear to the initial annotater from syntactic context. Only these unambiguous tokens are included in the analysis in section 3.1; all tokens are included in all other analyses.

A token was discarded if it probably contained a dysfluency or if the position of either the AA or the PA was obscured and not easily inferable from their surroundings or from their positions in the surrounding frames. Fewer than five percent of the originally identified tokens were excluded for these reasons. In total, 
3075 tokens representing 229 different sign types/lexemes were included in the analysis.

A small parallel experiment was carried out to collect subjective frequency ratings for each of the signs analyzed. We identified each head or neck sign occurring at least twice in the videos of the first five signers analyzed. One of the authors (a Deaf native signer) was recorded performing the canonical version of each sign type. Six signers from the Winnipeg Deaf community were asked to view the video clips of the signs in random order and rate each one for how often they thought they used that sign or saw it used by others, on a scale from 1 (almost never) to 7 (at least once a minute). These ratings were entered into a mixed effects model with no fixed effects and with lexeme and rater as random effects; the resulting coefficients for lexeme are used as our measure of subjective frequency.

\section{Results and discussion}

We will first see if we can replicate some of the effects found in earlier studies: the difference between nouns and verbs in Section 3.1 and the effect of lexical frequency in Section 3.2. We then turn to the questions of whether the lowering patterns that exist for signs canonically made on the forehead generalize to other locations on the face and neck, in Section 3.3, and whether sign lowering is completely gradient, completely categorical, or partially both, in Section 3.4. We finish by discussing the implications of the patterns in our corpus for the nature of undershoot, articulatory reduction, and phonological representations.

\subsection{Nouns vs. verbs}

Figure 2 shows the amount of lowering for verbs compared to nouns. It can easily be seen that the attainment points of a sizeable proportion of verbs are substantially lower than their canonical PA, while nouns are for the most part clustered tightly around their canonical PAs.

As in other studies, the difference between nouns and verbs is significant. In a mixed effects model with lexical category as the fixed effect and sign type/lexeme and signer as random effects, verbs are vertically displaced an extra -7.9 pixels compared to nouns $\left(t=-3.14, p_{t}=.0017, p_{M C M C}=.0001\right)$, while nouns' average displacement of -4.8 pixels is not significantly different from 0 .

The vertical difference between nouns and verbs is perhaps most striking in morphologically related noun/verb pairs. Figure 3 compares the attainment points of two signs with identical canonical PAs at the forehead: the verb TEACH and the noun TEACHER, which consists of TEACH plus a suffix (or compound 


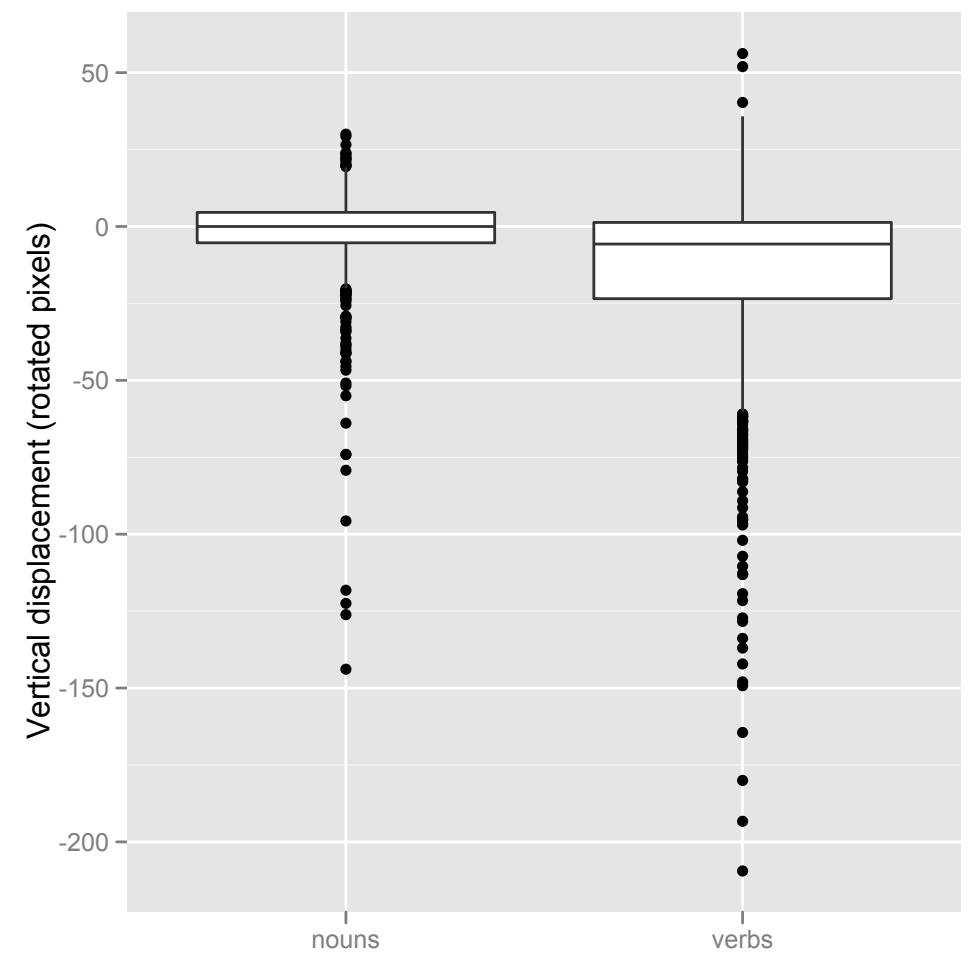

Figure 2: Degree of sign lowering in nouns and verbs

TEACHER

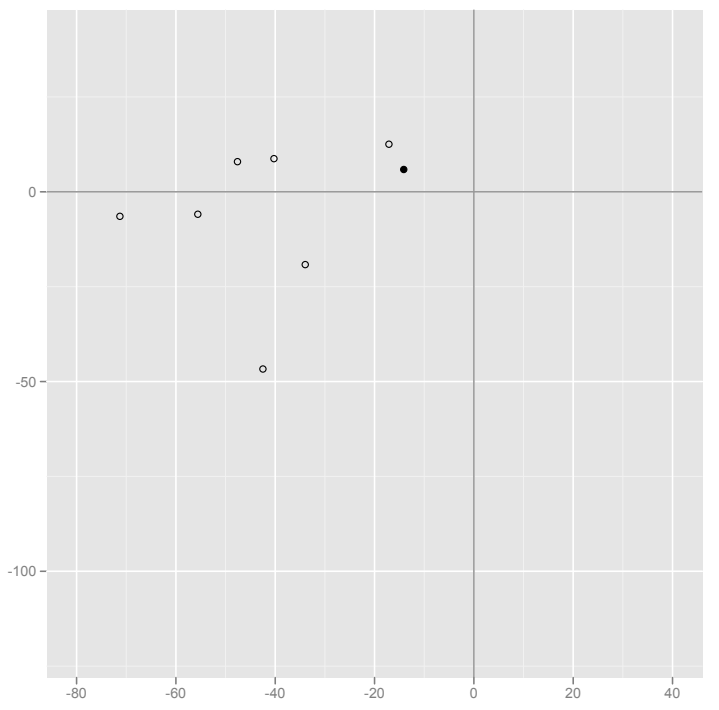

TEACH

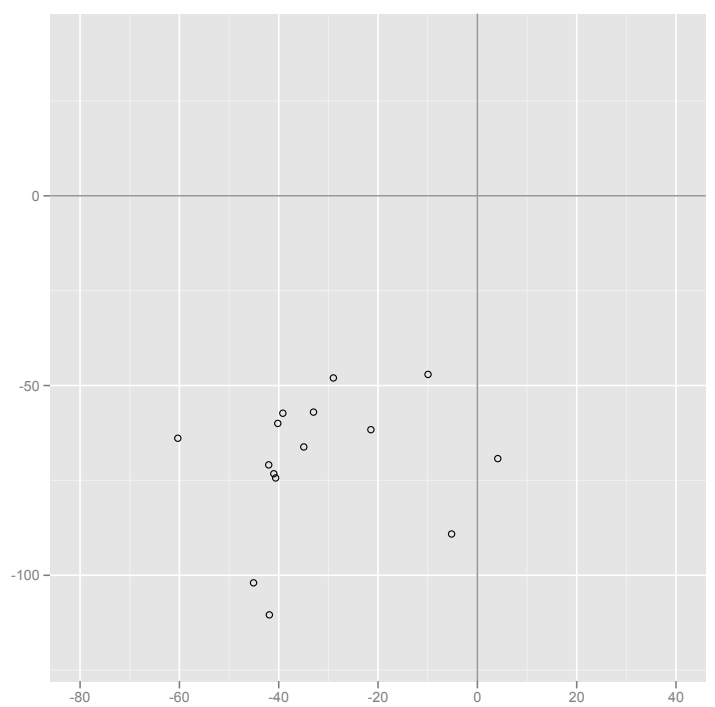

Figure 3: Difference in the attainment points between the noun TEACHER and the verb TEACH.

These figures, and all similar figures in the rest of the paper, are drawn to the same scale. Axes represent the (rotated) horizontal and vertical positions relative to the canonical passive articulator at the origin $(0,0)$; measurements are in pixels. Each circle represents one token of the sign type: filled circles indicate that the token contacted the signer's body, unfilled circles indicate no contact. 


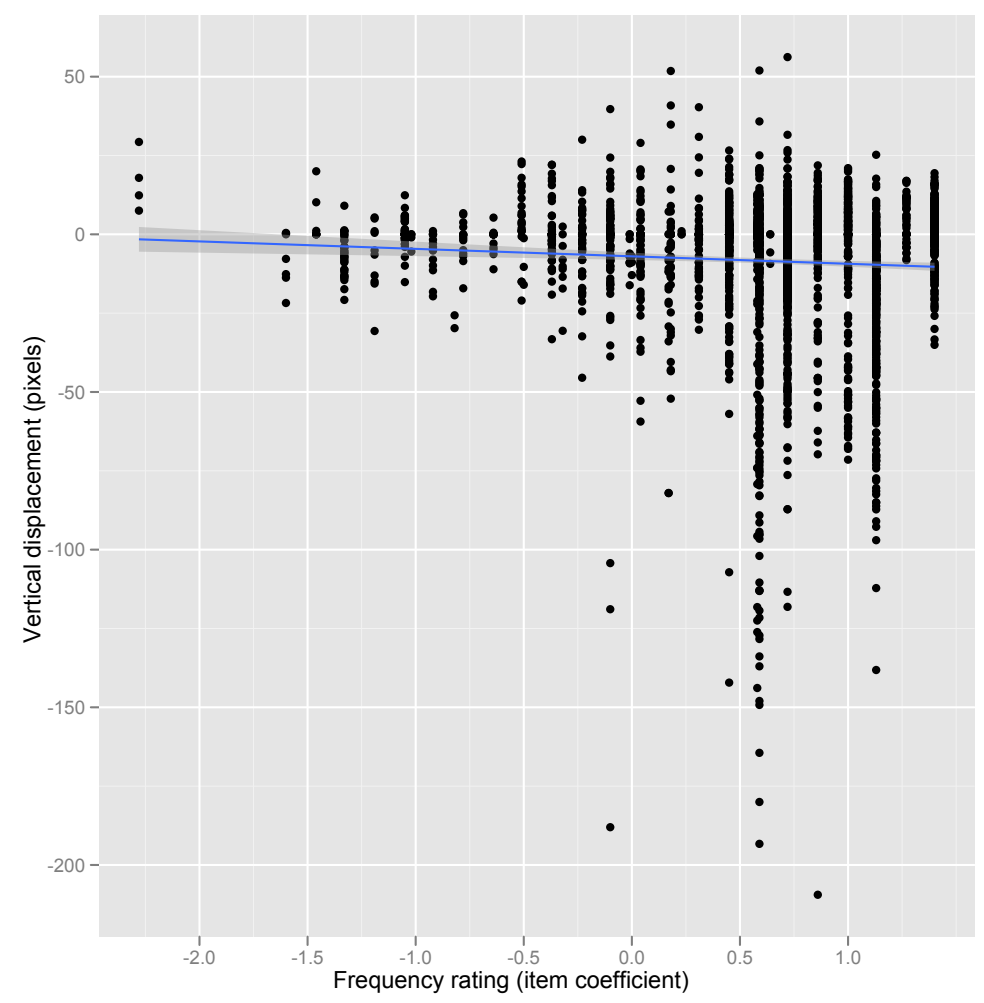

Figure 4: Effect of (subjective) lexical frequency on lowering.

head) indicating an agent. Figure 3 also explains the graphing conventions that are used in future figures.

Other lexical categories - adjectives, particles (prepositions, conjunctions, etc.), and negated verbs are intermediate, showing more lowering than nouns and less than verbs, but not significantly different from either.

\subsection{Lexical frequency}

Figure 4 shows the relationship between the lexical frequency of the signs and the amount of vertical displacement from their canonical PA. The effect is small, but significant.

In a mixed effects model predicting vertical displacement from subjective frequency, with signer and type/lexeme as random effects, the coefficient is -4.5 pixels per unit of subjective frequency on the sevenpoint scale $\left(t=-2.54, p_{M C M C}=0.0014\right)$. The effect of frequency remains significant in a model including general PA region (e.g., forehead, nose, chin) and lexical category as predictors $(\beta=-4.4, t=-2.43$, $\left.p_{M C M C}=0.0026\right)$.

There is no significant interaction between lexical category and frequency, such as the one Schembri et al. 


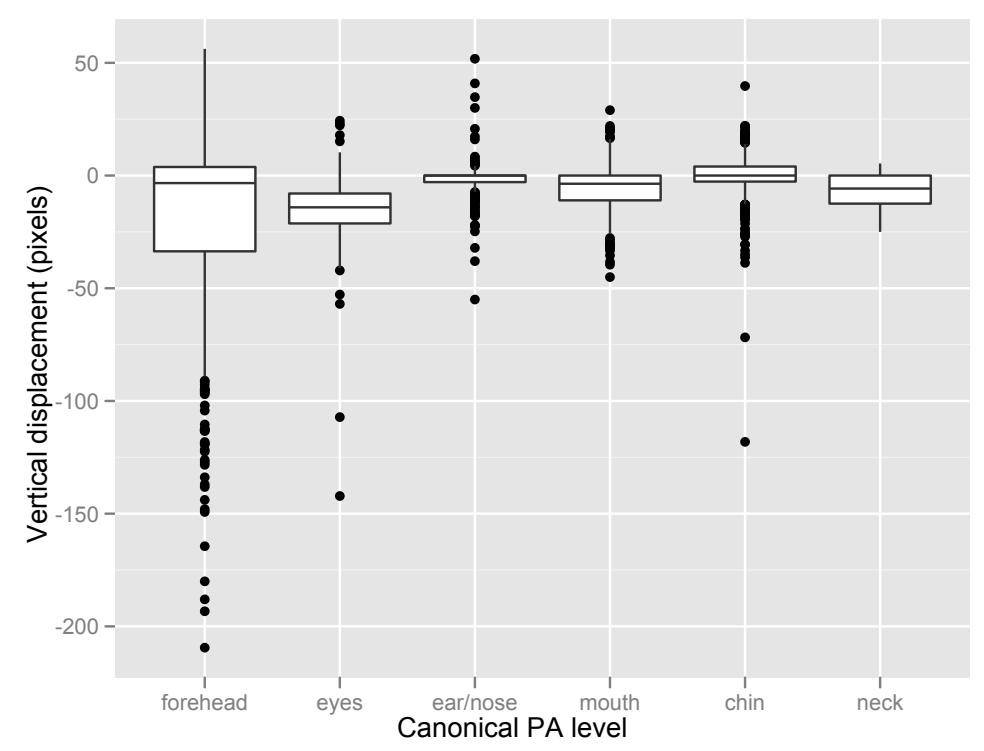

Figure 5: Degree of sign lowering according to the vertical level of the canonical PA.

(2009) found in New Zealand Sign Language, where high-frequency verbs were more likely to lower than low-frequency verbs, but high-frequency nouns and adjectives were less likely to lower than low-frequency nouns and adjectives. In our ASL data, nouns, like verbs, increasingly lower with increasing frequency.

\subsection{The effect of canonical location}

Previous studies have considered only signs that are canonically formed at the forehead. If sign lowering really is caused mainly by the mechanical difficulty of quickly moving the hand high in the signing space, we would expect to see the degree of sign lowering progressively lessen as the canonical PA moves down the face from the forehead to the eyes, nose, mouth, chin, and neck, as it presumably becomes progressively easier to reach the target.

Figure 5 partially confirms this expectation. We see a lot of lowering for forehead signs, less lowering for signs canonically formed relative to the eyes, and a great deal less lowering for signs formed at the level of the nose or ear, the mouth, the chin, or the neck.

The difference between forehead signs and lower signs is well illustrated by considering pairs of otherwise identical signs differing in the gender of the referent and therefore, for ASL, in canonical location: the male signs are canonically formed with contact on the forehead, the female signs with contact at the mouth or chin level. Figure 6 shows that tokens of SISTER are clustered reasonably tightly around their target, while 
BROTHER

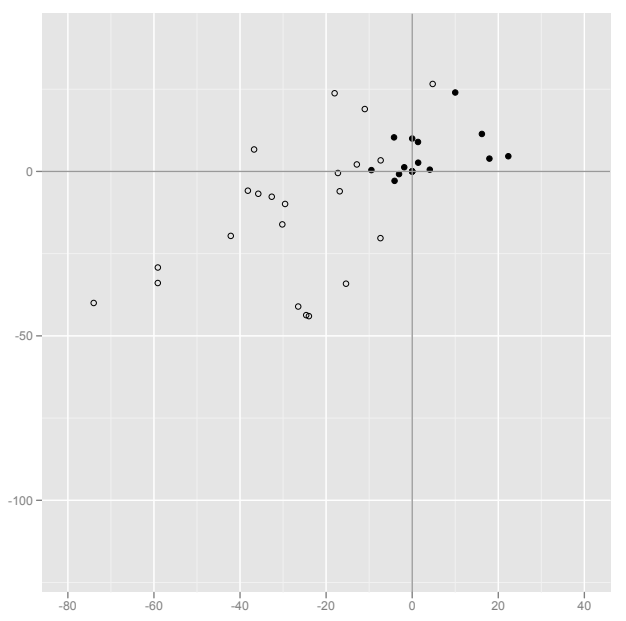

SISTER

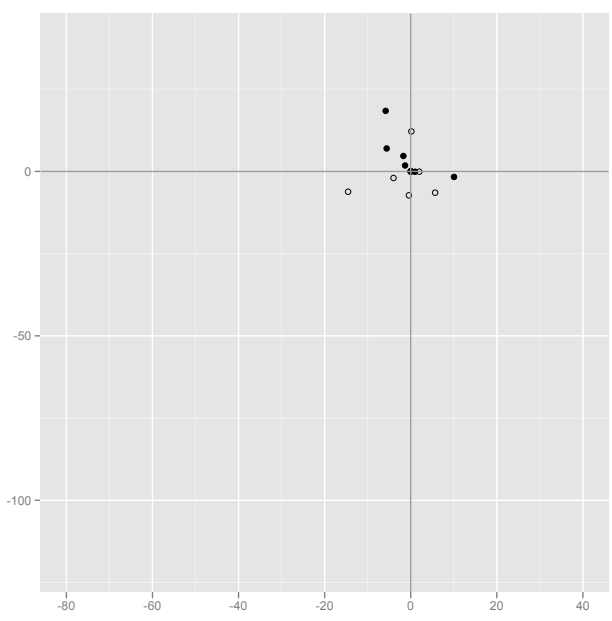

Figure 6: Attainment points for an almost identical pair of signs: BROTHER is formed th the forehead, SISTER at the chin.

those for the otherwise identical forehead sign BROTHER has a comparable cluster of tokens contacting near the target, but also a tail of other tokens (some contacting, more not) trailing off downwards and to the signer's right from the target. The pairs MOTHER/FATHER and MAN/WOMAN follow the same pattern in our corpus.

On the other hand, our expectation that the height of a sign's canonical PA should be gradiently related to its degree of lowering isn't completely borne out. While the forehead and eye levels fit nicely, the lower four levels show small and seemingly arbitrary degrees of lowering in Figure 5: signs canonically formed at the mouth and neck levels have small tendencies to lower, but signs canonically formed at the nose/ear level and at the chin level are no more likely to lower than to raise, despite being higher and presumably harder to reach than lower locations (the mouth and neck respectively) that encourage more sign lowering.

The fact that forehead signs undergo lowering substantially more than signs canonically formed at lower levels can't simply be blamed on the fact that the hand has a longer distance to travel in order to reach the forehead. Figure 7 redraws Figure 5, showing vertical position relative to the signer's right eye (at 0 ) rather than vertical displacement relative to each sign's canonical PA. We can see that, even in absolute terms, forehead signs show more extreme lowering than others. If signs canonically formed at the nose can consistently reach at least the level of the mouth, it is hard to see any simple mechanical reason why a forehead sign shouldn't be able to get at least that high as well.

Unfortunately, it seems that conclusions about undershoot based solely on studying signs canonically 


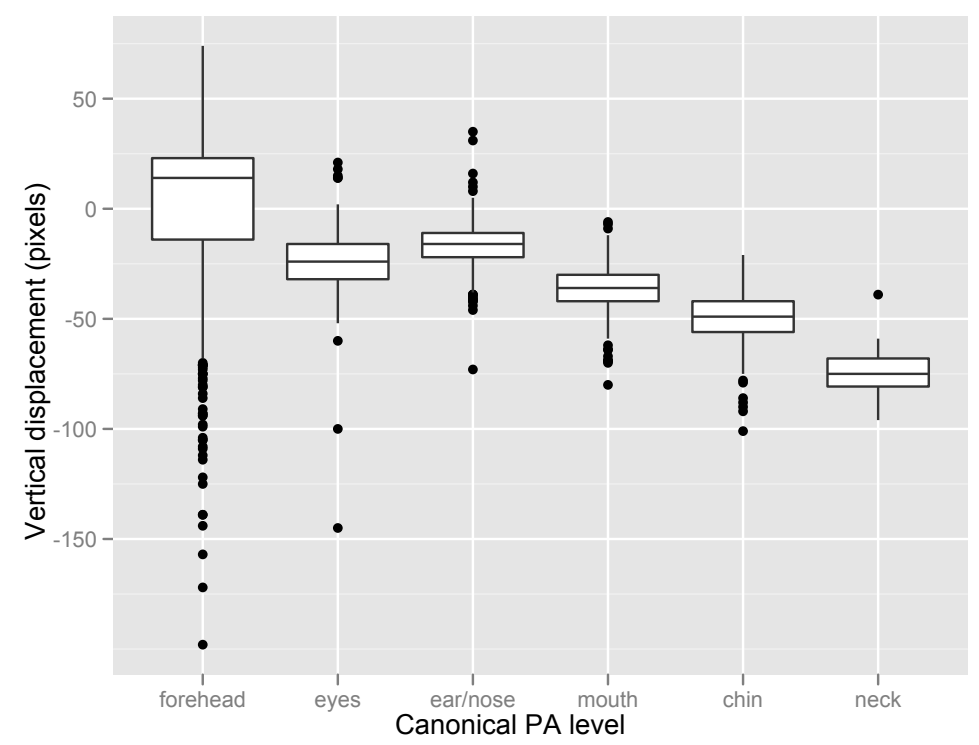

Figure 7: Absolute vertical position of the attainment point, grouped according to vertical level of the canonical $\mathrm{PA}$, but plotted relative to the signer's right eye $(=0)$.

formed at the forehead do not generalize in a straightforward way to signs formed at other locations on the head and neck.

\subsection{Is lowering categorical?}

One of the central questions of this paper is whether ASL sign lowering is a gradient process resulting from undershoot, as argued by the phoneticians, or a categorical process choosing between or creating two or more discrete phonological representations, as tacitly assumed in the variationist analyses.

Some signs really do seem to have their tokens distributed in two fairly discrete clusters in a way that suggests a categorical process, for example, KNOW (Figure 8). One cluster of tokens is close to KNOW's canonical PA on the forehead above the right eye, with a small tail of undershot non-contacting tokens trailing down and to the signer's right in a manner reminiscent of the male signs in Figure 6. A second cluster of tokens are formed lower, on the signer's cheek, sometimes contacting, sometimes not. While it seems natural to treat the first cluster's tail as failures to reach a forehead target under time pressure, it seems much less natural to treat the second cluster (especially the ones that contact the face slowly and deliberately) that way, so something other than mere undershoot is going on.

But, if KNOW seems an obvious candidate for a categorical analysis, other signs are more troublesome. DON'T-KNOW is comparable to KNOW in most relevant respects: its canonical PA is identical (as for 


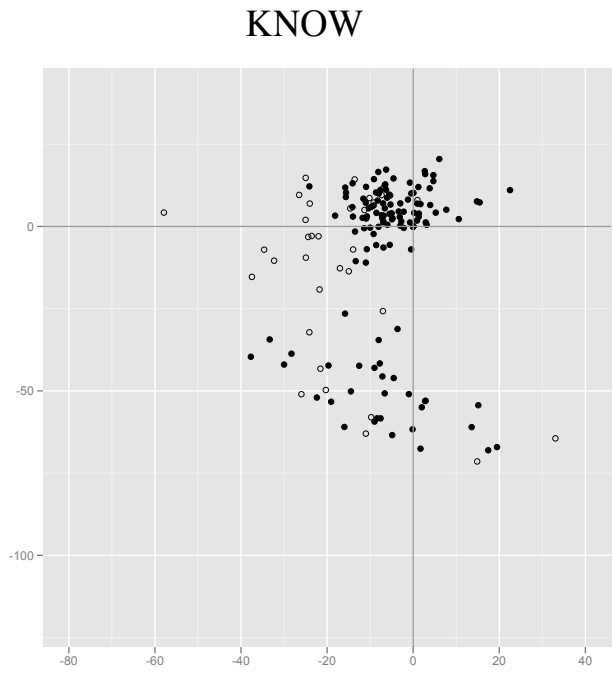

FOR
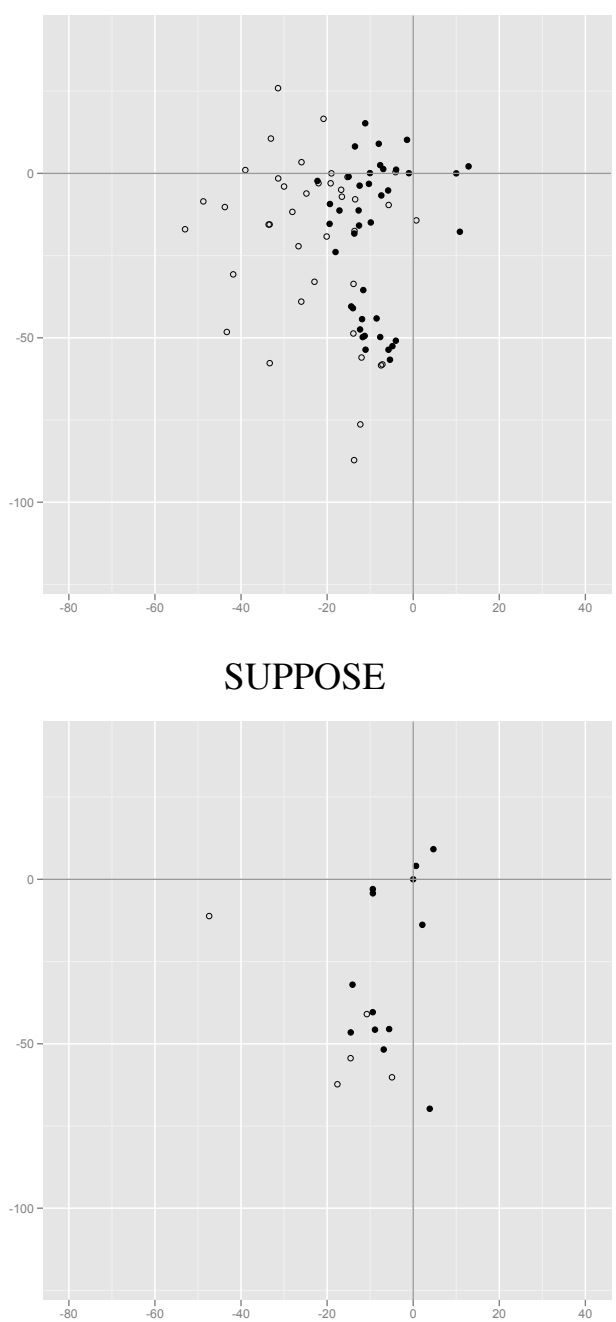

DON'T-KNOW

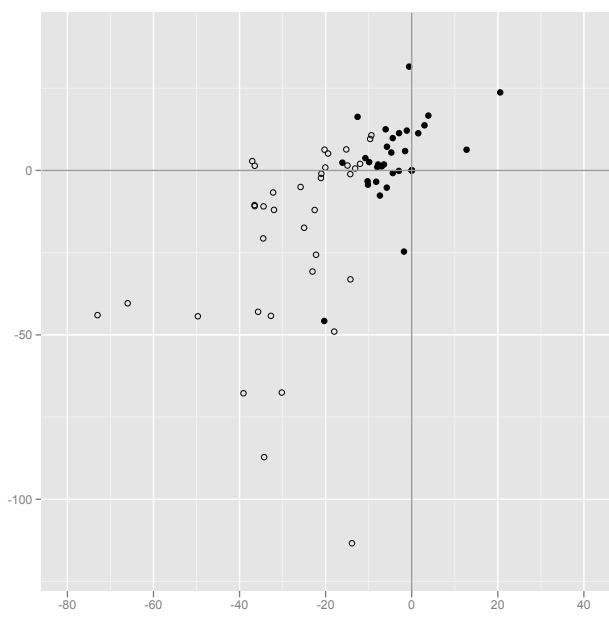

WHY

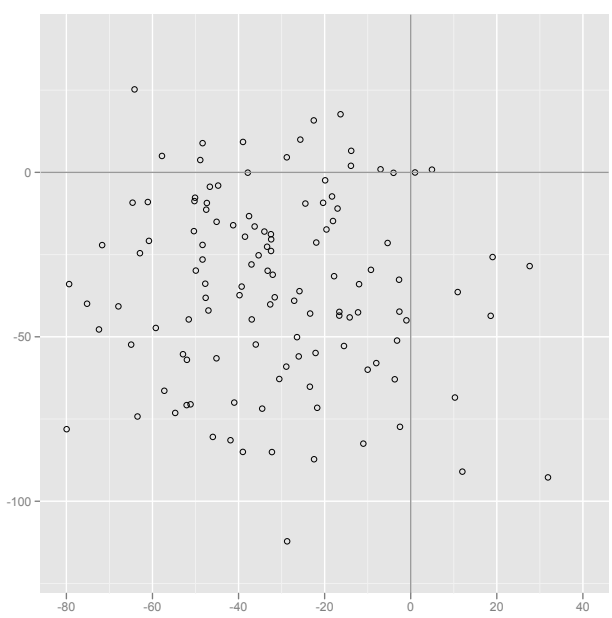

NOT

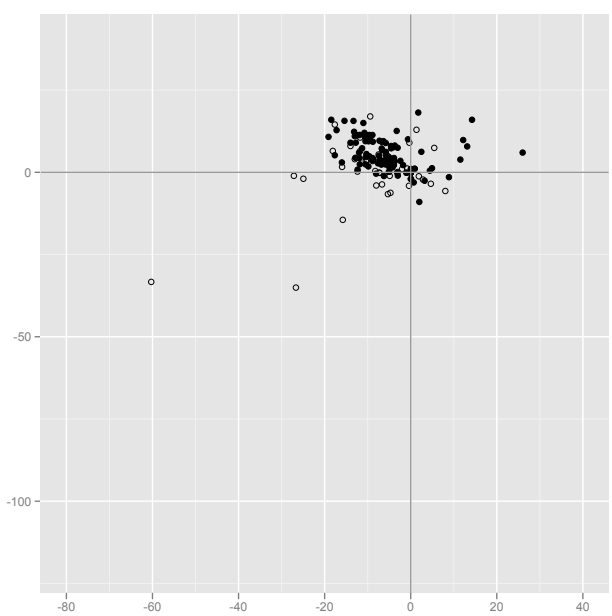

Figure 8: Attainment points of some signs discussed in the text. 
KNOW, the hand contacts the forehead above the right eye, but then twists outward for the negation), its frequency is close (72 tokens of DON'T-KNOW to 170 of KNOW in the corpus, only 0.28 less on the sevenpoint subjective frequency scale). Yet, as illustrated in Figure 8, while DON'T-KNOW has an even wider range of attainment points than KNOW, there is no compelling evidence for a second cluster of DON'TKNOW tokens, even though a couple of tokens do contact the signer's cheek, as for the lower cluster of KNOW tokens.

The answer to the question of whether sign lowering is a categorical process seems to be: sometimes, but usually not. Undershoot alone cannot explain the presence of more than one discrete cluster in the minority of signs like KNOW - these seem to require multiple targets as lexically specified variants. But multiple targets cannot begin to explain the attainment points of most tokens that are vertically or laterally displaced from their canonical PAs: the long tails of tokens in the male signs in Figure 6, the similar tails within each cluster of KNOW, or the huge, homogeneous cloud of attainment points for WHY (Figure 8). These seem to call for a gradient process, and undershoot is the most plausible candidate.

\subsection{Implications for undershoot}

The forehead sign FOR (Figure 8) illustrates the not-infrequent difficulty of determining whether a sign has one or two clusters of attainment points. On the one hand, FOR might be like KNOW, but with a stunted lower cluster. On the other hand, the separation between the two apparent clusters is modest - it looks almost as if a single continuous cluster had had its central members pushed to either side by some need to avoid the region vertically about 30 pixels below the forehead PA and horizontally in line with the signer's eye. Little reflection is needed to realize that the occupant of this forbidden zone is, in fact, the signer's eye.

This pattern isn't a peculiarity of the sign FOR. Figure 9 shows the attainment point of every token in the corpus that makes contact with the signer's body, all plotted relative to the signer's right eye at the origin $(0,0)$. Figure 9 strikingly illustrates the rather obvious fact: Signers systematically avoid poking themselves in the eye.

As trivial as this observation seems, the systematic avoidance imposes some important constraints on the nature of the motor planning system responsible for undershoot. This is another fact that multiple targets alone can't explain. Gradient undershoot (in at least a mild form) clearly exists within the clusters that would be associated with a single target; it has been clearly demonstrated by phonetic studies discussed in section 1.1; and it needs to be constrained. No matter how imprecise the attainment point may be within a 


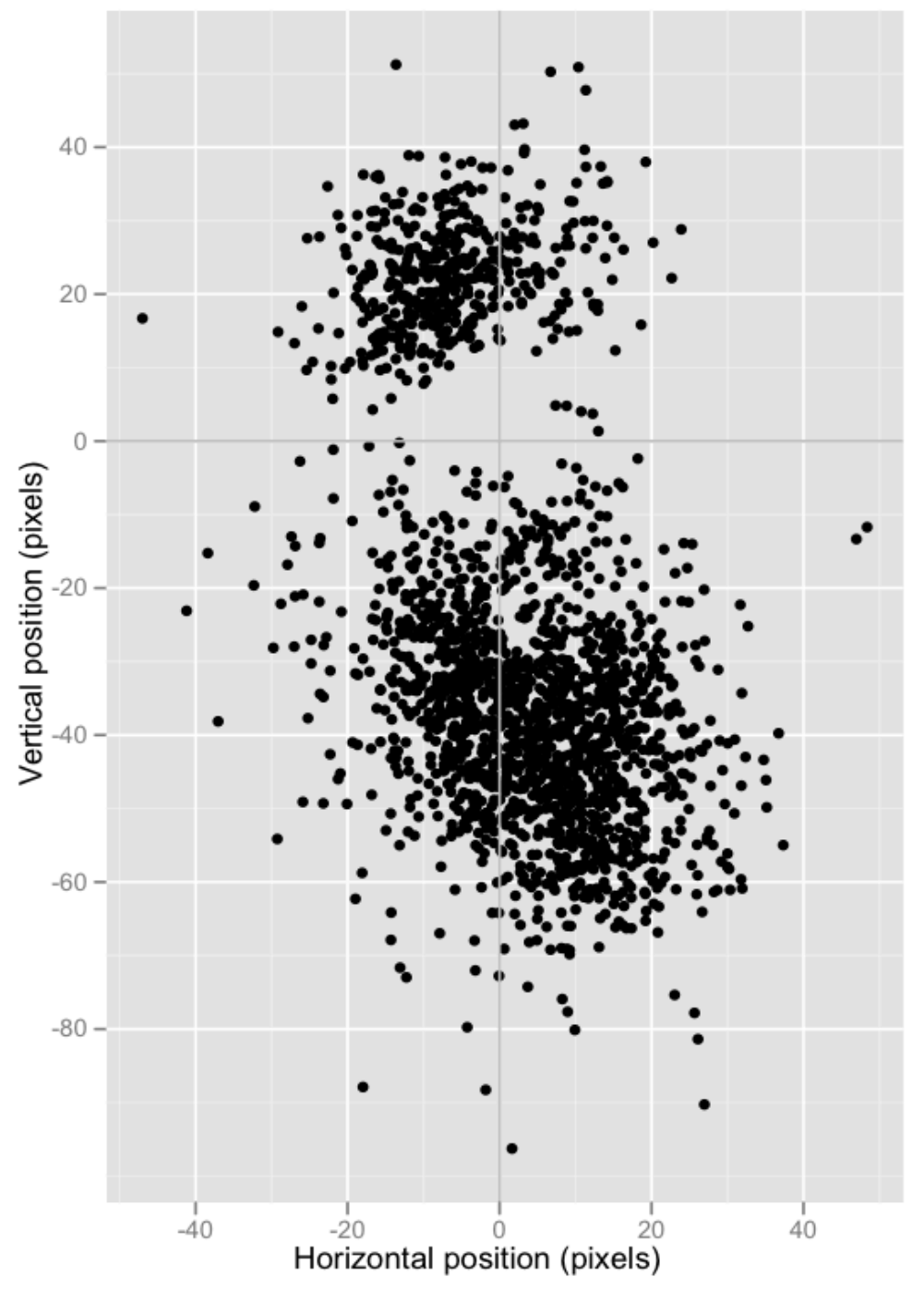

Figure 9: The attainment points of all tokens in the corpus that contacted the signer's body - all relative to the signer's right eye at the origin $(0,0)$. 
cluster, no matter how far undershot a target may be, the signer's gesture will never result in them poking themselves in the eye. ${ }^{3}$

The implication is that undershoot cannot be just the fully automatic side-effect of two gestures overlapping and pulling the same articulator in different directions. Rather, there must be some highly sophisticated forward modelling mechanism that predicts what the (specifically somatosensory) consequences of a particular degree of gestural overlap would be, and it predicts these consequences in time to veto any that would be painful. Undershoot is not accidentally missing the target - it is carefully planned and controlled.

The conclusion that sign-lowering undershoot is planned fits well with the arguments of Whalen (1990) that coarticulation in spoken languages is largely planned. It also fits well with an independent line of evidence on the importance of somatosensory feedback in sign production. Perceptual loop theory claims that the way speakers monitor themselves is to pass the acoustic consequences of their productions back through their speech recognition system (e.g., Postma 2000; Huettig \& Hartsuiker 2010). Emmorey et al. (2009) find that signers' recognition systems are very bad at coping with the kind of visual feedback they would get from their own productions, yet signers show all the same evidence of self-monitoring that speakers show, so Emmorey et al. conclude that self-monitoring in signers must rely mainly on somatosensory feedback rather than visual feedback. The most plausible way somatosensory feedback could be used in self-monitoring would be for the signer to compare it against the predicted somatosensory consequences from the same forward modelling mechanism we are arguing for.

\subsection{Reduction and phonological representations}

We echo Tyrone \& Mauk (2010) that it would be useful to consider ASL articulatory reduction in dimensions other than the vertical. But even restricting our attention to the vertical dimension, there is no easy equation between lowering and reduction once we consider more than just the forehead. Signs canonically made on the neck do lower a lot, often to contact the shoulder or upper chest, just as we would expect if lower locations were easier to reach. We would expect it to take even more effort for the hand to reach the nose than the neck, yet signs canonically formed on the nose show strikingly little lowering.

For some signs, articulatory ease (and therefore reduction) is actually served better by raising the sign, for example, NOT and DEAF. ${ }^{4}$ This would seem to suggest that the acceptable methods of reduction and acceptable ranges of attainment points depend in highly complicated ways on the precise combination of canonical location, contact, and movement type - perhaps even on the individual sign lexemes themselves 
(i.e., word-specific phonetics, cf. Pierrehumbert 2002). Establishing whether such sign-specific effects on reduction exist would require controlling for all the other factors that are known to affect reduction in signed and spoken languages, which would require a much larger corpus than ours and systematic coding of all the relevant variables. But in our initial impressions based on the current data, it seems rather unlikely that any combination of across-the-board variables will be able to fully predict the vast spread of WHY, the tight clustering of NOT, apparently under-motivated differences like the two-clusteredness of KNOW as opposed to DON'T-KNOW, or, even among signs with two fairly obvious clusters, explain why the upper cluster is relatively tight for KNOW and loose for FOR, while the lower cluster is loose for KNOW and tight for FOR.

Even if an adult signer had no need for explicitly represented knowledge of these sign-specific distributional oddities and could produce them automatically as the result of some complex combination of general factors, it is hard to see how the learner could figure out what that complex combination of factors is without first remembering specific distributions. This implies that, at least for learners, phonological representations refer directly to distributions over phonetic parameters. Exemplar models or hybrid abstract/exemplar models offer one straightforward account for this, but other distribution-based theories of phonological categories would also be up to the task (except those that assume all distributions can be defined with an extremely small set of parameters, such as those governing the Gaussian distribution). A distribution-based theory also makes it easy to imagine how similar distribution-based accounts of sociophonetic variation in spoken languages could be extended to handle the social factors that the variationist studies discussed in the introduction found to influence lowering in signed languages. See Pierrehumbert (2003) and Griffiths et al. (2008) for just two examples of the kinds of models that could handle the patterns we find.

We argued earlier that there is a somatosensory forward modelling mechanism responsible for avoiding undesirable contacts and for generating the expectations that are matched against feedback during monitoring. One plausible hypothesis is that the decisions of this modelling mechanism get fed back and incorporated into the learner's phonological representations. The on-line mechanism must still remain vigilant for the rest of the signer's life to make sure that undershoot never does any damage, but for the most part phonology will eventually stop asking for the kinds of gestural scores that would pose a danger.

For signs canonically formed by contacting the forehead, modifying the phonological distributions to take into account the vetoes of the forward modeller will usually result in distributions with clusters above and below the eye, i.e., distributions that look like they also could have been produced by a categorical lowering process. Signs canonically formed below eye-level (like NOT or SISTER) or signs formed at the 
forehead without contact (like WHY) will normally remain unaffected by the vetoes and have no discernible separation of clusters.

If our analysis is correct, then ASL sign lowering stands as one more piece of evidence that apparently categorical behaviour can sometimes be produced by inherently continuous processes under special conditions - an idea that phonologists are increasingly coming to terms with (e.g., Gafos \& Benus 2006; Wedel 2007; Scobbie \& Pouplier 2010).

\section{Conclusion}

In extending the study of ASL lowering beyond the forehead to all signs formed on the face and neck, we found partial support for analyses from both the phonetic and the variationist traditions discussed in the introduction. Like the phoneticians, we found clear evidence of target undershoot across locations, gradiently influenced in the expected way by lexical frequency. But, in keeping with the tacit variationist assumption of a categorical process, a small minority of signs had realizations that clearly fell into two clusters, and it was hard to see how the wide range of vertical displacements across different locations could be due to purely mechanical factors. There really is something different about forehead signs, something that can occasionally give at least the appearance of a categorical lowering process.

One of the most plausible explanations for the patterns we found goes as follows. A signer about to produce a sign will draw a target location from the phonological distribution in the sign's entry in the mental lexicon and incorporate that target into the gestural score they are building for the entire utterance. A forward modelling mechanism predicts the somatosensory consequences of that gestural score, vetoes it if it would result in an undesirable contact, and otherwise provides the signer's monitoring mechanism with something to compare their somatosensory feedback to. Over the course of learning, the patterns of successful vs. vetoed targets will become incorporated into the phonological distributions, resulting in the mental lexicon having "built in" to it the acceptable ranges of undershoot for each sign and some patterns that mimic the results of categorical processes.

No matter how accurate or inaccurate it is to see articulatory reduction as a form of physical laziness, mentally it takes just as much work as careful speech or signing, if not more. 


\section{Notes}

${ }^{1}$ We gratefully acknowledge all the signers whose recordings are analyzed here and who participated in the frequency rating mini-experiment; to Peter Lawford and Sherra Hall for technical support and performing many of the measurements; the editors of this special issue, Ian Maddieson and Caroline Smith, and three reviewers; and audience members at the 2010 Canadian Linguistics Association meeting, the Trends in Sign Language Research conference, and at LabPhon 10 for useful discussion — especially to Martha Tyrone, our discussant at LabPhon.

${ }^{2}$ In the absence of an agreed-upon and readable way of transcribing ASL signs, the tradition in sign language linguistics has been to represent a sign by printing the closest English translation equivalent (or gloss) in capital letters. In this paper, hyphens in an English gloss do not represent morpheme boundaries in ASL.

Video clips of the citation form of all signs discussed in this paper are available in the supplementary online materials.

${ }^{3}$ We probably shouldn't say that signers, even adult signers, never poke themselves in the eye. But the motor planning system for signed language is at least as successful at preventing it as the motor planning system for spoken language is at preventing speakers from accidentally biting their tongues.

Incidentally, signers apparently find it easy to generalize the avoidance constraint beyond just their eyeballs. None of our four signers who wore glasses ever produced a token that contacted their lenses, even if the lenses were large enough to extend below the canonical PA of a few signs on the cheekbone.

${ }^{4}$ In NOT, canonically formed by the thumb forward from the underside the chin, lowering is virtually absent and the most common displacement is upward so that the thumb tip contacts the front of the chin (Figure 8). This is arguably an easier location for the thumb to contact, though there is no obvious explanation for why the extremely high-frequency NOT (unlike, say, FOR) is so insistent on contacting the face at all. (We can't appeal to the functional load of unambiguously signalling negation, considering that NOT is usually accompanied by non-manual markers that can and often do signal negation perfectly well even in the absence of the manual sign NOT.) The form of DEAF used most often in our corpus canonically involves a contact by the index finger beside the corner of the mouth followed by a contact in front of the ear. (The less-used form of DEAF has the contacts in the opposite order.) Signers' reductions of DEAF seem focused on shortening the length of the movement between those two contacts by making them closer to each other, which involves raising the first contact higher than mouth-level. Indeed, a handful of tokens of DEAF have been reduced to a single contact on the cheek halfway between mouth and ear. 


\section{References}

Browman, Catherine P. \& Louis Goldstein. 1992. Articulatory Phonology: an overview. Phonetica 49. $155-180$.

Cheek, Adrianne. 2001a. The phonetics and phonology of handshape in American Sign Language: University of Texas at Austin dissertation.

Cheek, Adrianne. 2001b. Synchronic handshape variation in ASL: evidence of coarticulation. In Minjoo Kim \& Uri Strauss (eds.), NELS 31: Proceedings of the North East Linguistic Society, Georgetown University, volume one, 117-129. Amherst, MA: Graduate Linguistics Students' Association, University of Massachusetts.

Cormier, Kearsy. 2002. Grammaticization of indexic signs: how American Sign Language expresses numerosity: University of Texas at Austin dissertation.

Emmorey, Karen, Rain Bosworth \& Tanya Kraljic. 2009. Visual feedback and self-monitoring of sign language. Journal of Memory and Language 61(3). 398-411.

Gafos, Adamantios I. \& Stefan Benus. 2006. Dynamics of phonological cognition. Cognitive Science 30(5). 905-943.

Griffiths, Thomas L., Adam N. Sanborn, Kevin R. Canini \& Daniel J. Navarro. 2008. Categorization as nonparametric bayesian density estimation. In Nick Chater \& Mike Oaksford (eds.), The probabilistic mind: prospects for Bayesian cognitive science, 303-328. Oxford: Oxford University Press.

Huettig, Falk \& Robert J. Hartsuiker. 2010. Listening to yourself is like listening to others: external, but not internal, verbal self-monitoring is based on speech perception. Language \& Cognitive Processes 25(3). $347-374$.

Lucas, Ceil, Robert Bayley, Mary Rose \& Alyssa Wulf. 2002. Location variation in American Sign Language. Sign Language Studies 2(4). 407-440.

Mandel, Mark. 1981. Phonotactics and morphophonology in American Sign Language: University of California, Berkeley dissertation. 
Mauk, Claude E. 2003. Undershoot in two modalities: evidence from fast speech and fast signing: University of Texas at Austin dissertation.

Mauk, Claude E., Björn Lindblom \& Richard P. Meier. 2008. Undershoot of ASL locations in fast signing. In Josep Quer (ed.), Signs of the time: selected papers from TISLR 8, 3-24. Seedorf: Signum Verlag.

Mauk, Claude E. \& Martha Tyrone. 2008. Sign lowering as phonetic reduction in American Sign Language. In Proceedings of the 8th International Seminar on Speech Production, 185-188.

Öhman, S.E.G. 1966. Coarticulation in CVC utterances: spectrographic measurements. Journal of the Acoustical Society of America 39. 151-168.

Pierrehumbert, Janet. 2002. Word-specific phonetics. In Carlos Gussenhoven \& Natasha Warner (eds.), Laboratory Phonology 7, 101-139. Berlin: Mouton de Gruyter.

Pierrehumbert, Janet. 2003. Phonetic diversity, statistical learning, and the acquisition of phonology. Language and Speech 46(2-3). 115-154.

Postma, Albert. 2000. Detection of errors during speech production: A review of speech monitoring models. Cognition 77.97-131.

Schembri, Adam, David McKee, Rachel McKee, Sara Pivac, Trevor Johnston \& Della Goswell. 2009. Phonological variation and change in Australian and New Zealand Sign Languages: The location variable. Language and Variation and Change 21.193-231.

Scobbie, James M. \& Marianne Pouplier. 2010. The role of syllable structure in external sandhi: An EPG study of vocalisation and retraction in word-final English /1/. Journal of Phonetics 38(2). 240-259.

Sloetjes, Han, Albert Russel, Alex Klassmann, Peter Wittenburg \& Hennie Brugman. 2006. ELAN: a professional framework for multimodality research. In Proceedings of LREC 2006, Fifth International Conference on Language Resources and Evaluation, 1556-1559.

Tyrone, Martha E. \& Claude E. Mauk. 2010. Sign lowering and phonetic reduction in American Sign Language. Journal of Phonetics 38. 317-328.

Wedel, Andrew B. 2007. Feedback and regularity in the lexicon. Phonology 24(1). 147-185.

Whalen, D.H. 1990. Coarticulation is largely planned. Journal of Phonetics 18. 3-35. 